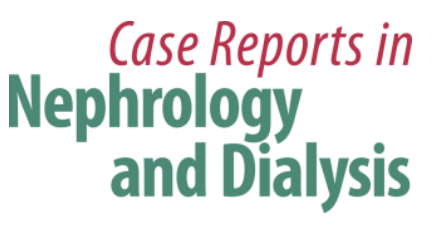

Case Rep Nephrol Dial 2016;6:70-75

\title{
Protracted Clinical Course of Postinfectious Glomerulonephritis in a Previously Healthy Child
}

\author{
Camilla Grøndahl $^{a} \quad$ Søren Rittig ${ }^{a} \quad$ Johan Vestergaard Povlsen ${ }^{b}$ \\ Kostantinos Kamperis ${ }^{a}$ \\ Departments of ${ }^{a}$ Pediatrics and ${ }^{b}$ Renal Medicine, Aarhus University Hospital, Skejby, \\ Aarhus, Denmark
}

\section{Key Words}

Acute postinfectious glomerulonephritis · Haematuria · Proteinuria $\cdot$ Complement C3

\begin{abstract}
Acute postinfectious glomerulonephritis (PIGN) affects children typically after upper respiratory tract or skin infections with streptococci but can complicate the course of other infections. In children, it is generally a self-limiting disease with excellent prognosis. This paper reports a previously healthy 4-year-old boy who experienced a protracted course of PIGN with persisting episodes of gross haematuria, proteinuria, decreased complement C3c levels but normal P-creatinine levels. Due to the protracted course and the nephrotic-range proteinuria, a renal biopsy was performed 6 months after the initial presentation and the overall pathology was consistent with acute endocapillary glomerulonephritis.
\end{abstract}

(C) 2016 The Author(s)

Published by S. Karger AG, Basel

\section{Introduction}

PIGN typically affects children between 4 and 12 years and the latency period after infection may vary from 1 to 2 weeks after pharyngitis, skin or other infections. The clinical findings are markedly variable, ranging from asymptomatic cases to severe, symptomatic forms [1]. Cardinal symptoms are sudden appearance of haematuria, proteinuria, oedema, hypertension and variable degree of renal insufficiency. PIGN in children is generally a selflimiting disease with excellent prognosis [2]. A small fraction of patients have persistent proteinuria and eventually progressive loss of kidney function leading to end-stage renal

\section{KARGER}

Camilla Grøndahl

Aarhus University School of Medicine

Department of Pediatrics, Aarhus University Hospital, Skejby

Paludan Müllersvej 101, DK-8210 Aarhus V (Denmark)

E-Mail camilla.groendahl@gmail.com 


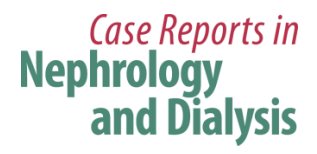

Grøndahl et al.: Protracted Clinical Course of Postinfectious Glomerulonephritis in a Previously Healthy Child

failure $[3,4]$. We herein describe a case of PIGN with protracted course in a previously healthy child.

\section{Case Description}

A 4-year-old child with no past medical history presented with recurrent episodes of macroscopic haematuria for 3 weeks. The boy did not complain of lower urinary tract symptoms and clinical examination was unremarkable with no oedemas and normal blood pressure. Laboratory investigations revealed reduced P-albumin of $33 \mathrm{~g} / \mathrm{l}$ (35-47 g/l), normal Pcreatinine, P-urea and electrolytes. Urine albumin excretion at presentation was $57 \mathrm{mg} /$ day based on 24-hour urine collection. Complement C3c was low at $0.37 \mathrm{~g} / \mathrm{l}(0.68-1.27 \mathrm{~g} / \mathrm{l})$ and C4 was normal. Anti-nuclear, anti-neutrophil cytoplasmic antibodies (ANCA) and C3 nephritic factor were all negative. Anti-streptococcal antibody titers were moderately elevated ( $>400$ units). Based on the clinical and laboratory findings, PIGN was suspected and the child was discharged to outpatient follow-up.

During the following months, the boy presented with several episodes of gross haematuria, fatigue and recurrent periorbital oedema. Some of the episodes were related to upper respiratory tract infections. Laboratory workup revealed gradually increasing proteinuria (up to $2.4 \mathrm{~g} /$ day) and decreasing P-albumin $(24 \mathrm{~g} / \mathrm{l})$ but normal P-creatinine. Plasma complement C3c varied between 0.40 and $0.66 \mathrm{~g} / \mathrm{l}$ (fig. 1). Due to the protracted course and the nephrotic-range proteinuria, a renal biopsy was performed 6 months after the initial presentation showing endocapillary proliferation and characteristic subepithelial deposits (humps) in the basement membrane but no crescents. The renal tubules, interstitium, and vessels were spared. No immune deposits or C3 staining was seen and the overall pathology was consistent with PIGN.

A year after the initial presentation, the boy still presented with persistent proteinuria ( $0.6 \mathrm{~g} /$ day) and decreased P-albumin $(29 \mathrm{~g} / \mathrm{l})$ levels, but normal P-creatinine. Complement C3c was persistently decreased $(0.38 \mathrm{~g} / \mathrm{l})$. Treatment with ACE inhibitor (ramipril) was initiated approximately 40 weeks after onset and the proteinuria decreased subsequently to $0.3 \mathrm{~g} /$ day.

\section{Discussion}

Clinical symptoms indicative of an acute glomerulonephritis in a child should raise the suspicion of PIGN but other types of glomerulonephritis leading to a similar clinical presentation may include mesangioproliferative IgA nephritis, lupus nephritis, ANCA-associated vasculitis (AAV), and membranoproliferative glomerulonephritis (MPGN). The activation of complement can help differentiate between these conditions. Low plasma complement factor C3c level is indicative but not pathognomonic for PIGN since MPGN and lupus nephritis may also be associated with hypocomplementaemia. The boy was negative for anti-nuclear and ANCA screening and had normal levels of complement C4 [3]. A renal biopsy, although rarely indicated in PIGN, can be used to rule out other diagnoses.

Recent data suggest a possible role for the complement system in atypical cases of PIGN as functional and genetic studies of the alternative pathway identified autoantibodies or mutations in complement genes in such patients. Positivity for C3 nephritic factor was also associated with other functional abnormalities of the alternative pathway [5]. 


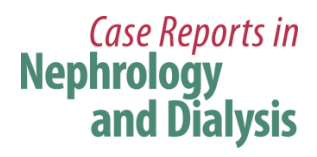

Case Rep Nephrol Dial 2016;6:70-75 \begin{tabular}{l|l}
\hline DOI: $10.1159 / 000445678$ & $\begin{array}{l}\text { (c) } 2016 \text { The Author(s). Published by S. Karger AG, Basel } \\
\text { www.karger.com/cnd }\end{array}$
\end{tabular}

Grøndahl et al.: Protracted Clinical Course of Postinfectious Glomerulonephritis in a Previously Healthy Child

Dense deposit disease (DDD) and C3 glomerulonephritis (C3GN) are classified as 'C3 glomerulopathies' [6]. Both diseases are a result of abnormal regulation of the alternative complement pathway, leading to excessive activation, a process that may be induced by C3 nephritic factor (C3NeF) or be the result of genetic variation affecting the complement system [6]. C3NeF is an IgG autoantibody that directly stabilizes the C3-activating complex of the alternative pathway, thus preventing the normal inhibitory action of complement factor $\mathrm{H}$. Examples of genetic causes include gain-of-function mutations in genes that encode proteins that activate the alternative pathway, and loss-of-function mutations in genes that encode regulators of the alternative pathway [7]. C3NeF is found in approximately $40-50 \%$ of patients with C3GN and in approximately $80 \%$ of patients with DDD [8]. Reduced serum C3 levels and normal C4 levels are consistent with alternative pathway dysregulation, which can help us distinguish C3 glomerulopathies from other types of glomerulonephritis, but is neither sensitive nor specific for the diagnosis of C3 glomerulopathies [9]. In many cases of PIGN, the circulating levels of C3 are typically low, indicating an activation of the alternative pathway, but in the majority of cases, this is a self-limiting form of C3 glomerulopathy because the serum C3 returns to normal once the infection resolves [6]. Subepithelial 'humps' that are identical to those seen in PIGN may be identified in both DDD and C3GN [6]. Due to this knowledge, atypical PGIN can be difficult to distinguish from a C3 glomerulopathy [10].

Screening for genes currently known to be involved in the pathogenesis of alternative pathway disorders may be considered when a diagnosis of DDD, C3 glomerulopathy or atypical PIGN is established or suspected [11]. The most common genes implicated are CFH, CFI, CD46, CFHR1-5, CFB and C3 [7, 11]. In the present case, the boy was negative for C3NeF and a genetic analysis as well as a repeat biopsy may be helpful in establishing a definite diagnosis.

Treatment of PIGN remains mainly supportive. Early antibiotic therapy may reduce the period of bacterial antigen exposure but has not been proven to prevent PIGN. Treatment of ongoing streptococcal infections is generally advised. Fluid overload may be treated with sodium restriction and diuretics [3]. Control of hypertension is essential to reduce morbidity. In severe cases with acute renal failure, renal replacement therapy may be necessary [12]. In endemic or epidemic courses, early antibiotic treatment can prevent the spread of the infection and prophylactic antibiotic treatment can be initiated to individuals at risk [13]. In case of histologic evidence of crescents in a renal biopsy, indicating a greater degree of acute inflammation, high-dose corticosteroids have been used. Generally, however, there is no evidence that corticosteroids are beneficial in treating PIGN [3]. PIGN in children is generally a self-limiting disease with excellent prognosis [2], but persistent haematuria or proteinuria have been reported in up to $20 \%$ of PIGN patients at 10 years of follow-up [3].

Complement C3 is an important component of the pathogenesis of PIGN and is decreased in more than $90 \%$ of all cases. This decrease tends to occur even before the development of clinical symptoms and persists for up to 8 weeks [14]. In the present case, the levels of plasma C3c varied between 0.38 and $0.66 \mathrm{~g} / \mathrm{l}$ and were still decreased 1 year after onset. The persisting low level of plasma C3c is a sign of activated alternative complement pathway and is associated with disease activity. Proteinuria tends to resolve shortly after plasma creatinine levels return to baseline within 3 to 4 weeks [3]. In the present case, the boy had increasing proteinuria during the following 6 months after onset, whereas plasma creatinine remained within normal range.

Renal biopsy is typically not necessary to confirm the diagnosis of PIGN but is indicated in cases with atypical course, such as prolonged decrease in plasma C3c, recurrence of gross haematuria, progressive increase in proteinuria, and deterioration of renal function [14]. The biopsy in our case showed endocapillary proliferation, characteristic subepithelial de- 
Grøndahl et al.: Protracted Clinical Course of Postinfectious Glomerulonephritis in a Previously Healthy Child

posits (humps) in the basement membrane and no crescents, which is thus fully consistent with PIGN (fig. 2).

Some of the episodes of gross haematuria were associated with symptoms of either upper respiratory tract infection or gastroenteritis. One may speculate that his recovery period was extended by such episodes of viral upper respiratory tract infection and gastroenteritis, or by a reinfection with a streptococcal infection during the hypocomplementaemic phase.

Previous studies have shown an association between human leukocyte antigen antigens and the incidence of PIGN. There is ongoing research of the putative streptococcal antigen involved in the immune complex formation. Nephritis-associated streptococcal plasmin receptor is a plasmin-binding protein with glyceraldehyde-3-phosphate dehydrogenase activity and has been in focus as a possible nephritogenic factor [12].

In a recent study, some patients with 'atypical' PIGN share an underlying defect in the regulation of the alternative complement pathway [5]. In this study, 'atypical' PIGN included cases of persistent haematuria and proteinuria that showed features of PIGN in kidney biopsies, but without evidence of a preceding infection. Even after resolution of the acute symptoms, the complement levels were not normalized and haematuria and proteinuria persisted. The identified gene variations included mutations in complement regulation antibodies and proteins in the $\mathrm{C} 3$ [5].

PIGN is generally a self-limiting form of glomerulonephritis with activation of the alternative complement pathway as the main pathophysiological background. Complement activity returns to normal typically after 6-8 weeks when the infection resolves. Patients with atypical PIGN can share clinical and pathologic abnormalities with C3 glomerulopathies. A defect in the regulation of the alternative complement pathway may explain the atypical forms of PIGN, and genetic tests can distinguish between the two.

\section{Take-Home Messages}

- $\quad$ PIGN is generally a self-limiting disease with good prognosis, but cases with protracted course exist and such a case is presented.

- $\quad$ The persisting low level of plasma C3c is a sign of activated alternative complement pathway and is associated with disease activity.

- $\quad$ Renal biopsy may be needed to rule out other diagnoses.

- $\quad$ Patients with 'atypical' PIGN may have an underlying defect in the regulation of the alternative complement pathway.

\section{Statement of Ethics}

The authors have no ethical conflicts to disclose.

\section{Disclosure Statement}

There are no conflicts of interest to declare. 
Grøndahl et al.: Protracted Clinical Course of Postinfectious Glomerulonephritis in a Previously Healthy Child

\section{References}

1 Marques Vde P, Neves PD, Mendonca HM, Fugikaha I, Fernandes EL: Acute glomerulonephritis after upper airway or skin infection: descriptive analysis of 82 cases between 14 and 64 years-old. J Bras Nefrol 2010;32:237-240.

-2 Kasahra T, Hayakawa H, Okubo S, Okugawa T, Kabuki N, Tomizawa S, Uchiyama M: Prognosis of acute poststreptococcal glomerulonephritis (APSGN) is excellent in children, when adequately diagnosed. Pediatr Int 2001;43:364-367.

-3 VanDeVoorde RG 3rd: Acute poststreptococcal glomerulonephritis: the most common acute glomerulonephritis. Pediatr Rev 2015;36:3-13.

4 Clark G, White RH, Glasgow EF, Chantler C, Cameron JS, Gill D, Comley LA: Poststreptococcal glomerulonephritis in children: clinicopathological correlations and long-term prognosis. Pediatr Nephrol 1988;2:381-388.

5 Sethi S, Fervenza FC, Zhang Y, Zand L, Meyer NC, Borsa N, et al: Atypical postinfectious glomerulonephritis is associated with abnormalities in the alternative pathway of complement. Kidney Int 2013;83:293-299.

6 Cook HT, Pickering MC: Histopathology of MPGN and C3 glomerulopathies. Nat Rev Nephrol 2015;11:4-22.

-7 Fakhouri F, Bacchi VF, Noël LH, Cook HT, Pickering MC: C3 glomerulopathy: a new classification. Nat Rev Nephrol 2010;6:494-499.

8 Bomback AS, Appel GB: Pathogenesis of the C3 glomerulopathies and reclassification of MPGN. Nat Rev Nephrol 2012;8:634-642.

-9 Barbour TD, Pickering MC, Cook HT: Dense deposit disease and C3 glomerulopathy. Semin Nephrol 2013;33:493-507.

10 Sandhu G, Bansal A, Ranade A, Jones J, Cortell S, Markowitz GS: C3 glomerulopathy masquerading as acute postinfectious glomerulonephritis. Am J Kidney Dis 2012;60:1039-1043.

-11 Angioi A, Fervenza FC, Sethi S, Zhang Y, Smith RJ, Murray D, et al: Diagnosis of complement alternative pathway disorders. Kidney Int 2016;89:278-288.

12 Ahn SY, Ingulli E: Acute poststreptococcal glomerulonephritis: an update. Curr Opin Pediatr 2008;20:157162.

13 Rodriguez-Iturbe B, Musser JM: The current state of poststreptococcal glomerulonephritis. J Am Soc Nephrol 2008;19:1855-1864.

14 Lau KK, Wyatt RJ: Glomerulonephritis. Adolesc Med Clin 2005;16:67-85.

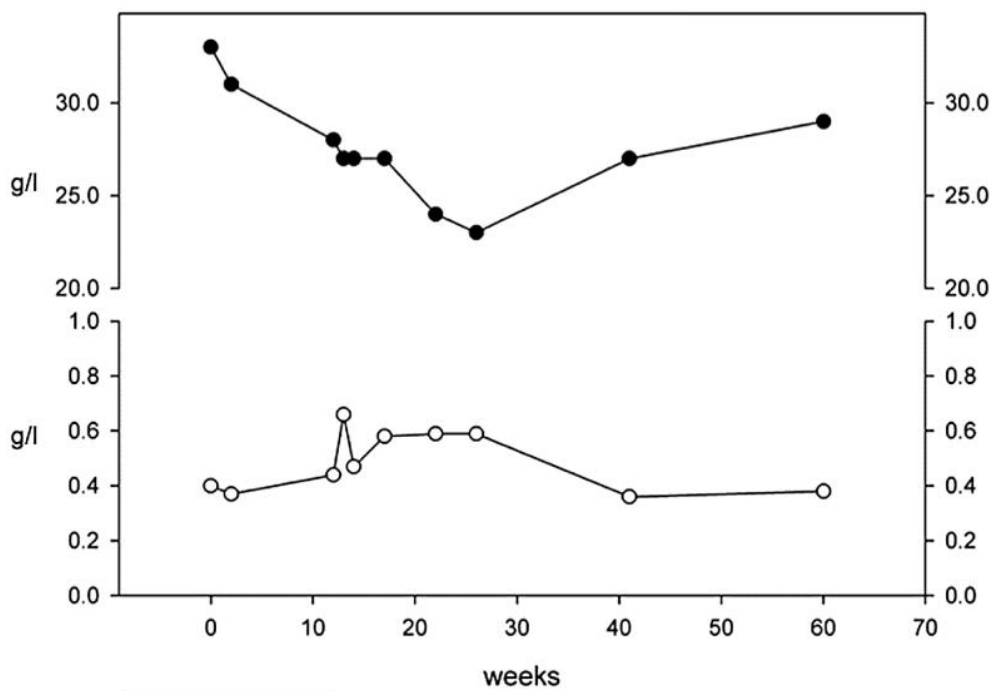

$$
\begin{aligned}
& \rightarrow-\text { Plasma Alb } \\
& \rightarrow-\mathrm{C} 3 \mathrm{c}
\end{aligned}
$$

Fig. 1. Plasma albumin (g/l) and plasma complement factor $\mathrm{C} 3 \mathrm{c}(\mathrm{g} / \mathrm{l})$ over time. 


\section{Case Reports in \\ Nephrology \\ and Dialysis}

\begin{tabular}{l|l}
\hline Case Rep Nephrol Dial 2016;6:70-75 \\
\hline DOI: 10.1159/000445678 & $\begin{array}{l}\text { C 2016 The Author(s). Published by S. Karger AG, Basel } \\
\text { www.karger.com/cnd }\end{array}$ \\
\hline
\end{tabular}

Grøndahl et al.: Protracted Clinical Course of Postinfectious Glomerulonephritis in a Previously Healthy Child
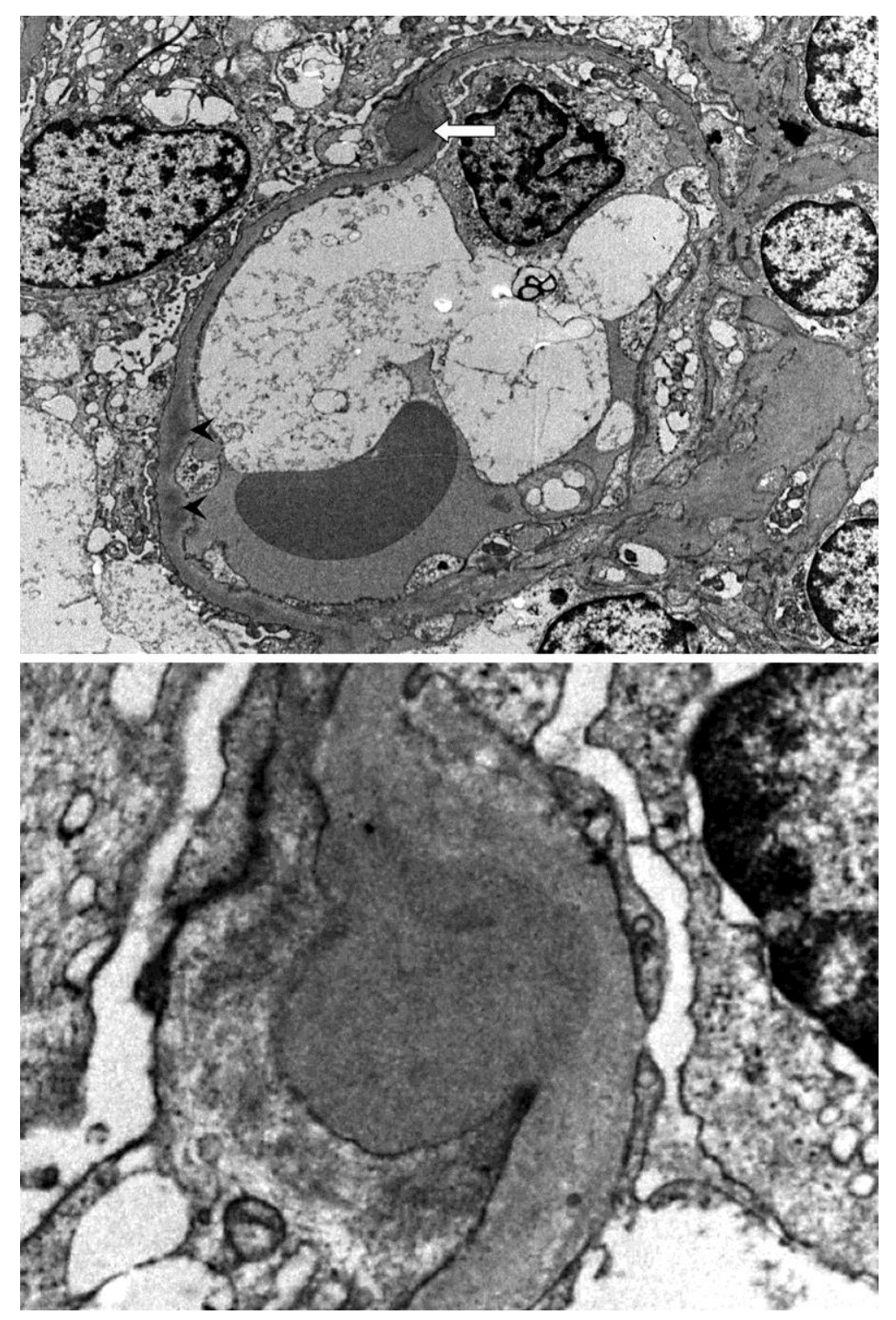

Fig. 2. Electron microscopic (EM) findings of the renal biopsy showing endocapillary proliferation and characteristic subepithelial deposits (humps) in the basement membrane. The humps are marked with arrows in the EM. No crescents were seen in the biopsy. Renal tubules, interstitium, and vessels were spared. No immune deposits or C3 staining was seen and the overall pathology was consistent with PIGN. 